\title{
Aplicações das dosagens de inibinas em Ginecologia e Obstetrícia
}

\author{
Clinical usefulness of inbibin assays in Gynecology and Obstetrics
}

Palavras-chave

Inibinas

Fertilidade

Gravidez

Keywords

Inhibins

Fertility

Pregnancy
Correspondência:

Fernando Marcos dos Reis Laboratório de Reprodução Humana Professor Aroldo F. Camargos Avenida Alfredo Balena $110,9^{\circ}$ andar (EP 310130-100 - Belo Horizonte (MG), Brosi Fone: (31) 3409-9485; Fax: (31) 3409-9299 E-mail: reis@medicina.ufmg.br

Recebido 15/10/09

\section{Resumo}

Na mulher, a principal fonte de inibina B são as células da granulosa de folículos em crescimento, enquanto a inibina A é secretada principalmente pelo corpo lúteo e pela placenta. Em mulheres inférteis submetidas a terapias de reprodução assistida, a inibina $B$ se mostrou útil para predizer má resposta ovulatória, embora não tenha superado o desempenho de outros marcadores. No rastreamento pré-natal da síndrome de Down, a utilidade da inibina A foi repetidamente confirmada no segundo trimestre e começa a ser considerada também na bateria de testes do primeiro trimestre. Além das duas aplicações acima, a dosagem de inibina total pode contribuir para a identificação de casos de insuficiência ovariana autoimune. A inibina total também pode ser um marcador auxiliar no diagnóstico de tumores epiteliais do ovário, enquanto a dosagem de inibina B auxilia no diagnóstico de tumores de células da granulosa. $\bigcirc$ uso da inibina A pode se estender à avaliação de gestantes com ameaça de abortamento, com história de abortamento de repetição, com risco aumentado de pré-eclâmpsia, ou ainda nos primeiros dias de seguimento pós-esvaziamento de mola hidatiforme. Todas essas aplicações continuam em estudo, mas com possibilidade real de virem a ampliar o espectro diagnóstico das dosagens de inibinas em Ginecologia e Obstetrícia.

\section{Abstract}

The main source of inhibin B in women is the growing follicle granulosa cells, while inhibin A is mainly produced by the corpus luteum and the placenta. In infertile women submitted to therapies of assisted reproduction, inhibin B has shown to be useful to predict a poor ovulatory response, though it has not yet overcome the performance of other markers. In the pre-natal screening of the Down syndrome, inhibin A has been repeatedly confirmed as useful in the second trimester and has also started to be considered in the first trimester test battery. Besides the two applications above, the dosage of total inhibin may contribute to the identification of cases of autoimmune ovarian insufficiency. Total inhibin may also be an auxiliary marker in the diagnosis of ovarian epithelial tumors, while the amount of inhibin B helps in the diagnosis of granulosa cells tumors. The use of inhibin A may be extended to the evaluation of pregnant women with risk of abortion, with a history of repeated abortion, with increased risk of pre-eclampsia, or even in the first days of follow-up of hydatiform mole post-emptying. All those applications are still under study, but with a real possibility of helping to extend the diagnostic spectrum of inhibin dosage in Gynecology and Obstetrics.

\footnotetext{
' Professor Adjunto do Departamento de Ginecologia e Obstetrícia da Universidade Federal de Minas Gerais - UFMG - Belo Horizonte (MG), Brasil.

2 Pós-graduanda (Mestrado) em Saúde da Mulher da Universidade Federal de Minas Gerais - UFMG - Belo Horizonte (MG), Brasil. Conflito de interesses: nenhum.
} 


\section{O que são inibinas e como atuam}

Inibinas são glicoproteínas da família do fator de crescimento TGF $\beta$, produzidas por diversos tipos celulares em vários tecidos e órgãos, com destaque para os ovários, testículos e placenta. As inibinas A e B são formadas por uma subunidade $\alpha$, comum a ambas, e uma subunidade $\beta$, que pode ser do tipo $\beta A$ (presente na inibina $A$ ) ou $\beta B$ (presente na inibina $B$ ). Atuam fundamentalmente como antagonistas das ativinas, com quem compartilham grande homologia estrutural, uma vez que a subunidade proteica beta das inibinas é a mesma que se combina em homodímero para dar origem às ativinas (Figura 1).

A atuação das inibinas decorre de sua competição pelo receptor tipo II de ativina. Para isso, as inibinas se valem da ligação entre sua subunidade $\alpha$ e um co-receptor chamado betaglicano, que é o mesmo receptor tipo III do TGF $\beta$ (Figura 1). Assim, as inibinas dificultam o acionamento da cascata de sinalização intracelular mediada pelas proteínas do grupo Smad e, consequentemente, reduzem ou bloqueiam os efeitos finais da ativina, tais como o estímulo à liberação hipofisária de $\mathrm{FSH}^{1}$.

\section{Como são feitas as dosagens de inibinas}

As primeiras dosagens de inibinas foram feitas por um radioimunoensaio inespecífico, desenvolvido em 1987, capaz de detectar indistintamente as formas diméricas ativas das inibinas $\mathrm{A}$ e $\mathrm{B}$ e seus precursores inativos, como a subunidade $\alpha$ isolada. $\mathrm{O}$ método não chegou à rotina clínica por causa de dificuldades técnicas relacionadas à imunorreatividade dos anticorpos empregados e devido ao surgimento, nove anos mais tarde, de uma nova geração de ensaios imunoenzimáticos, mais específicos e de

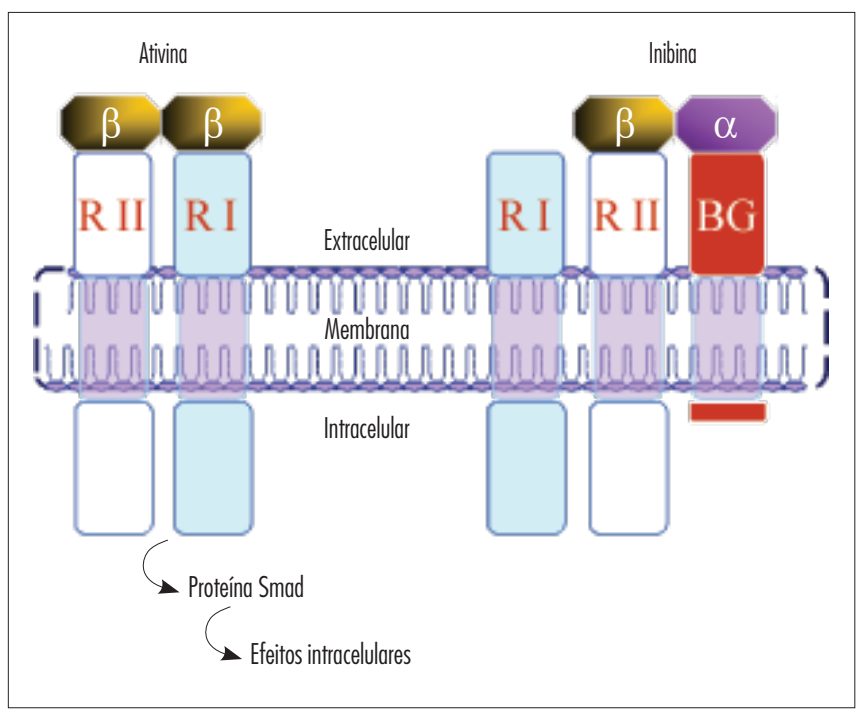

Figura 1 - Representação esquemática das inibinas (subunidades $\alpha+\beta$ ) e ativinas (subunidades $\beta+\beta$ ), receptores de ativina tipo I (RI) e tipo II (RII) e correceptor betaglicano (BG). muito mais fácil execução - portanto mais adequados ao uso comercial ${ }^{2}$.

Os ensaios imunoenzimáticos atuais (ELISA) usam um par de anticorpos monoclonais para capturar as duas subunidades das inibinas e permitem quantificar com precisão as concentrações de inibina $\mathrm{A}$ ou inibina $\mathrm{B}$ no soro e outros fluidos biológicos. O processo é semiautomatizado, semelhante a diversas outras dosagens feitas de rotina nos laboratórios clínicos, utiliza kits comercialmente disponíveis e o resultado é obtido em poucas horas ${ }^{3}$. Valores de referência foram estabelecidos para as diferentes fases do ciclo menstrual (para ambas as inibinas) e da gestação (para inibina A). Os coeficientes de variação se situam em torno de 4 a $8 \%{ }^{3}$.

\section{Aplicações diagnósticas em Ginecologia e Obstetrícia}

O uso diagnóstico das inibinas é baseado em uma série de constatações fisiológicas. Na mulher, a principal fonte de inibina B são as células da granulosa de folículos em crescimento $^{2}$, o que faz da inibina B um marcador de atividade folicular e reserva ovariana, com níveis mais altos na fase folicular do ciclo menstrual, redução na perimenopausa, níveis mínimos ou indetectáveis na pósmenopausa $^{3,4}$ e elevação considerável em caso de tumor das células da granulosa ${ }^{5}$. Quanto à inibina $\mathrm{A}$, suas fontes principais são o corpo lúteo e a placenta e, portanto, sua utilidade diagnóstica está ligada às diversas disfunções placentárias, como as associadas a abortamento precoce, cromossomopatias e pré-eclâmpsia ${ }^{2}$.

A dosagem de inibinas tem demonstrado, ao longo dos anos e dos diversos estudos realizados, várias aplicações potenciais em Ginecologia e Obstetrícia. Diversas dessas aplicações ainda permanecem em campo de pesquisa científica, mas algumas já puderam ser introduzidas na clínica (Quadro 1 e Tabela $1^{6,7}$ ).

Quadro 1 - Principais aplicações das dosagens de inibinas em Ginecologia e Obstetrícia. Aquelas assinaladas com 0 asterisco $(*)$ integram a rotina assistencial de diversos centros médicos, enquanto as demais poderão ser validadas e incorporadas no futuro.

Ginecologia (inibina B)
- Insuficiência ovariana primária
- Infertilidade e reserva ovariana*
- Tumores ovarianos


Tabela 1 - Considerações sobre o uso diagnóstico das dosagens de inibinas em Ginecologia e Obstetrícia

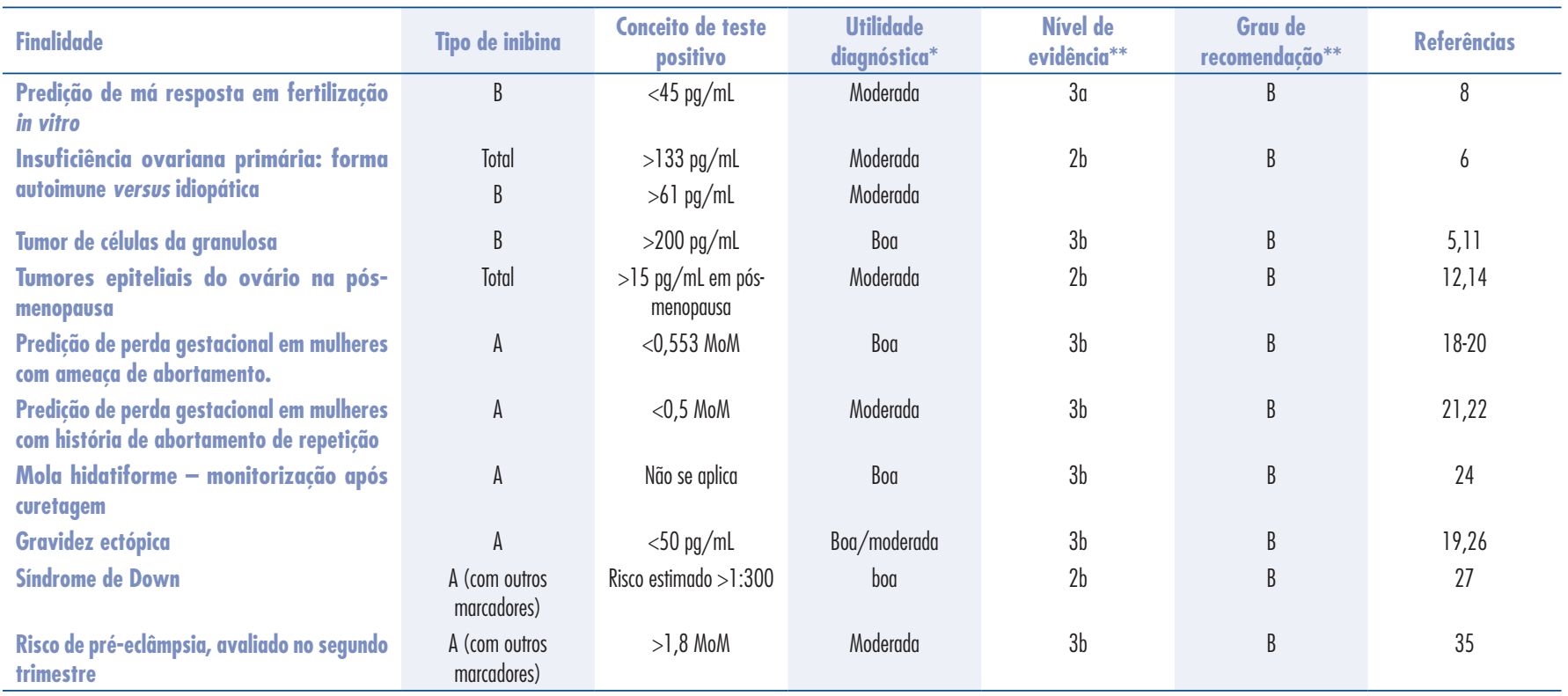

${ }^{*}$ Classificada de acordo com Khan et al. ${ }^{6} ;{ }^{*}$ Segundo os critérios do Centre for Evidence Based Medicine7; MoM: múltiplos da mediana.

\section{Aplicações em Ginecologia}

$\mathrm{Na}$ área de endocrinologia ginecológica, a transição para a menopausa se caracteriza por redução progressiva nos níveis séricos de inibina $B$, que precede e provoca a elevação dos níveis de $\mathrm{FSH}^{4}$. Assim como na transição para menopausa fisiológica, também nos casos de insuficiência ovariana primária observa-se redução dos níveis circulantes de inibinas ${ }^{8}$. Entretanto, quando a insuficiência ovariana é decorrente de ooforite autoimune, pelo menos na fase inicial parece ocorrer destruição preferencial das células da teca, com preservação das células da granulosa e, consequentemente, da produção de inibinas. Isso permite o diagnóstico diferencial da insuficiência ovariana autoimune (níveis normais ou altos de inibina total) daquela idiopática (níveis baixos de inibinas) ${ }^{8}$.

No campo da infertilidade masculina, já foi demonstrado que a contagem e concentração de espermatozoides no sêmen, assim como o volume testicular, apresentam correlação positiva com os níveis de inibina B e inversa com os de $\mathrm{FSH}^{9}$. Na propedêutica do casal infértil, outro aspecto de fundamental importância é a determinação da reserva ovariana. Diversos estudos já demonstraram inúmeros marcadores que poderiam ser empregados para estimar a função dos ovários, sendo o principal deles a idade da mulher ${ }^{10}$. A inibina B permanece como um dos marcadores de reserva ovariana mais estudados ${ }^{10,11}$. Porém, em estudos mais recentes, outros marcadores como o hormônio antimulleriano (AMH) apresentam correlação mais consistente com a reserva ovariana do que a dosagem de inibina $\mathrm{B}$ ou a contagem de folículos antrais ${ }^{11}$. Quando se avalia especificamente o prognóstico de pacientes submetidas à fertilização in vitro, a dosagem sérica de inibina B e AMH no dia da coleta ovular se correlacionam significativamente com o número de oócitos coletados ${ }^{12}$. Para a predição de gravidez após fertilização in vitro, o desempenho da inhibina B é modesto, mas o teste tem bom desempenho para a predição de má resposta, especialmente quando se encontram níveis inferiores a $45 \mathrm{pg} / \mathrm{mL}^{10}$.

Os níveis de inibinas também podem ter utilidade diagnóstica em oncologia ginecológica. Inibina B está aumentada em mulheres com tumores de células da granulosa de forma consistente, permitindo o diagnóstico com sensibilidade e especificidade próximos de $100 \%^{5,13,14}$. Dados preliminares sugerem que a expressão local de inibina pode ter alguma correlação com o risco de recorrência dos tumores de células da granulosa, embora o nexo fisiopatológico não esteja claro ${ }^{15}$. Além da elevação dos níveis séricos de inibina $\mathrm{B}$ em mulheres com tumor de células da granulosa, observa-se também aumento da inibina total em mulheres com carcinoma seroso ou mucinoso do ovário ${ }^{16}$. Assim, a dosagem de inibina total pode ser um marcador auxiliar, complementar ao CA-125 e mais sensível que este último, no diagnóstico de tumores epiteliais do ovário ${ }^{17}$.

No câncer de endométrio, o estudo da expressão tecidual das subunidades de inibina revelou que a subunidade $\alpha$ está diminuída nos tumores em comparação com o endométrio normal ${ }^{18}$. Dentre os tumores, aqueles positivos para subunidade $\alpha$ da inibina apresentam fatores prognósticos mais favoráveis, como grau histológico mais baixo, estadiamento cirúrgico menos avançado e menor proporção de casos com acometimento linfonodal ${ }^{19}$. Os casos positivos para subunidade $\alpha$ também se associam a 
maior sobrevida ${ }^{19}$. Esses achados sugerem que a presença da subunidade $\alpha$ da inibina é um fator de proteção contra a proliferação neoplásica do endométrio e que sua detecção por imuno-histoquímica contribui para a formulação do prognóstico ${ }^{18,19}$.

\section{Aplicações em Obstetrícia}

No início da gestação, a dosagem de inibina A pode auxiliar na predição de perda gestacional em mulheres com ameaça de abortamento. Diante de sangramento no primeiro trimestre caracterizado como ameaça de abortamento, níveis de inibina A abaixo de $50 \%$ dos valores correspondentes à mesma idade gestacional se associam a uma probabilidade aumentada de evolução para perda gestacional ${ }^{20-22}$. Dois estudos independentes também sugeriram que, em mulheres com história pregressa de abortamento de repetição e ainda assintomáticas, concentrações baixas de inibina A no primeiro trimestre sinalizam uma probabilidade aumentada de nova perda gestacional ${ }^{23,24}$. A dosagem de inibina $\mathrm{A}$ também é útil para monitorizar os primeiros dias pós-tratamento para mola hidatiforme, uma vez que sua redução é bem mais rápida que a do hCG, seja após quimioterapia ${ }^{25}$, seja após curetagem ${ }^{26}$.

A dosagem de inibina A pode auxiliar no diagnóstico de gestação ectópica. Pacientes com gravidez ectópica apresentam inibina A significativamente menor que pacientes com gestação eutópica, o que pode ser um dado valioso nos quadros de gestação de localização incerta ${ }^{21,27}$. Esse dado foi confirmado por um estudo independente que, porém, não demonstrou superioridade da inibina A sobre o hCG como indicador diagnóstico de gestação ectópica ${ }^{28}$.

Uma das utilizações mais importantes da dosagem de inibina A é no rastreamento da síndrome de Down. Tradicionalmente, o rastreamento bioquímico para essa síndrome é realizado com as dosagens séricas de alfafetoproteína, gonadotrofina coriônica humana e estriol, cujo período ótimo para realização é entre 16 e 18 semanas de gestação. Esse teste triplo pode ser aprimorado com o acréscimo da inibina $A$, gerando o teste quádruplo do segundo trimestre, que detecta cerca de $80 \%$ dos casos (contra 60\% do teste triplo). Quando se deseja antecipar o rastreamento para o fim do primeiro trimestre, pode-se utilizar o teste combinado que inclui a medida de transluscência nucal e as dosagens de proteína-A plasmática associada à gravidez (PAPP-A) e gonadotrofina coriônica humana (hCG). A combinação dessa bateria de testes do primeiro trimestre com o teste quádruplo do segundo trimestre permite detectar até $96 \%$ dos casos de síndrome de Down $^{29}$. Um estudo recente sugere que a dosagem de inibina A no rastreamento do primeiro trimestre contribui para elevar a sensibilidade do teste básico, composto por transluscência nucal, PAPP-A e hCG ${ }^{30}$.

Dentre as mais graves intercorrências em Obstetrícia se encontram as síndromes hipertensivas. Marcadores que podem ajudar a prever sua ocorrência ou prognóstico são de grande importância no seu manejo. Pacientes que apresentaram pré-eclâmpsia têm níveis mais altos de inibina $A$ do que mulheres que permaneceram normotensas durante a gravidez ${ }^{31}$. Níveis de inibina A no terceiro trimestre da gestação já foram correlacionados com a gravidade da pré-eclâmpsia em mulheres que tiveram o diagnóstico estabelecido nessa fase da gravidez. Quanto maiores os níveis da inibina A, maior a gravidade da doença ${ }^{32}$. No entanto, verifica-se considerável sobreposição dos níveis de inibina A nos diversos graus de pré-eclâmpsia, tornando sua dosagem pouco precisa para avaliar a gravidade do quadro ${ }^{33}$.

De maior relevância clínica seria poder predizer a ocorrência de pré-eclâmpsia em gestantes com fatores de risco para a doença, quando ainda se encontram assintomáticas. No primeiro trimestre de gestação, os níveis de inibina $\mathrm{A}$ já se encontram elevados em número significativo de mulheres que posteriormente desenvolvem a doença, mas o valor preditivo do teste é insuficiente para que ele seja levado em consideração ${ }^{34-36}$. Já no segundo trimestre, os níveis médios de inibina $\mathrm{A}$ já se mostram mais altos semanas antes do aparecimento da pré-eclâmpsia, mas seu valor preditivo positivo é baixo e não recomenda o seu uso como marcador isolado para predizer a doença ${ }^{36}$. Nessa fase, a dosagem de inibina $\mathrm{A}$ pode ser associada à de ativina $\mathrm{A}$ e à doplervelocimetria das artérias uterinas, melhorando assim a capacidade de predizer a ocorrência de pré-eclâmpsia na mesma gestação ${ }^{37}$.

\section{Referências}

1. Cook RW, Thompson TB, Jardetzky TS, Woodruff TK. Molecular biology of inhibin action. Semin Reprod Med. 2004;22(3):269-76.

2. Tsigkou A, Luisi S, Reis FM, Petraglia F. Inhibins as diagnostic markers in human reproduction. Adv Clin Chem. 2008;45(1): 1-29.

3. Cobellis L, Luisi S, Pezzani I, Reis FM, De Leo V, Petraglia F. Serum inhibin $A$, inhibin $B$, and pro-alphaC levels are altered after surgically or pharmacologically induced menopause. Fertil Steril. 2002;77(4): 745-9.

4. Sammel MD, Freeman EW, Liu Z, Lin H, Guo W. Factors that influence entry into stages of the menopausal transition. Menopause. 2009;16(6): 1218-27.

5. Petraglia F, Luisi S, Pautier P, Sabourin JC, Rey R, Lhomme $C$, et al. Inhibin $B$ is the major form of inhibin/activin family 
secreted by granulosa cell tumors. J Clin Endocrinol Metab. 1998;83(3): 1029-32.

6. Khan KS, Khan SF, Nwosu CR, Arnott N, Chien PF. Misleading authors' inferences in obstetric diagnostic test literature. Am J Obstet Gynecol. 1999;181(1):112-5.

7. Centre for Evidence Based Medicine [Internet]. Oxford, UK: CEBM; 2009 [cited 2009 Aug 16]. Available from: <http:// www.cebm.net>.

8. Tsigkou A, Marzotti S, Borges L, Brozzetti A, Reis FM, Candeloro P, et al. High serum inhibin concentration discriminates autoimmune oophoritis from other forms of primary ovarian insufficiency. J Clin Endocrinol Metab. 2008;93(4):1263-9.

9. Stewart TM, Liu DY, Garrett C, Jorgensen N, Brown EH, Baker $\mathrm{HW}$. Associations between andrological measures, hormones and semen quality in fertile Australian men: inverse relationship between obesity and sperm output. Hum Reprod. 2009;24(7): 1561-8.

10. Broekmans FJ, Kwee J, Hendriks DJ, Mol BW, Lambalk CB. A systematic review of tests predicting ovarian reserve and IVF outcome. Hum Reprod Update. 2006;12(6):685-718.

11. Riggs RM, Duran EH, Baker MW, Kimble TD, Hobeika E, Yin L, et al. Assessment of ovarian reserve with anti-Müllerian hormone: a comparison of the predictive value of anti-Müllerian hormone, follicle-stimulating hormone, inhibin B, and age. Am J Obstet Gynecol. 2008;199(2):202.el-8

12. Jee BC, Ku SY, Suh CS, Kim KC, Lee WD, Kim SH, et al. Serum anti-Müllerian hormone and inhibin $B$ levels at ovulation triggering day can predict the number of immature oocytes retrieved in in vitro fertilization cycles. J Korean Med Sci. 2008;23(4):657-61.

13. Mom CH, Engelen $\mathrm{N}$, Willemse PH, Gietema JA, ten Hoor KA, de Vries EG, et al. Granulosa cell tumors of the ovary: the clinical value of serum inhibin $A$ and $B$ levels in a large single center cohort. Gynecol Oncol. 2007; 105(2):365-72.

14. Robertson DM, Cahir N, Burger HG, Mamers P, Groome N. Inhibin forms in serum from postmenopausal women with ovarian cancers. Clin Endocrinol (Oxf). 1999;50(3):381-6.

15. Nosov V, Silva I, Tavassoli F, Adamyan L, Farias-Eisner R, Schwartz $P E$. Predictors of recurrence of ovarian granulosa cell tumors. Int J Gynecol Cancer. 2009;19(4):628-33.

16. Tsigkou A, Marrelli D, Reis FM, Luisi S, Silva-Filho AL, Roviello F, et al. Total inhibin is a potential serum marker for epithelial ovarian cancer. J Clin Endocrinol Metab. 2007;92(7):2526-31.

17. Robertson DM, Pruysers E, Jobling T. Inhibin as a diagnostic marker for ovarian cancer. Cancer Lett. 2007;249(1):14-7.

18. Florio P, Ciarmela P, Reis FM, Toti P, Galleri L, Santopietro R, et al. Inhibin alpha-subunit and the inhibin coreceptor betaglycan are downregulated in endometrial carcinoma. Eur J Endocrinol. 2005; 152(2):277-84.

19. Mylonas I, Worbs S, Shabani N, Kuhn C, Kunze S, Schulze S, et al. Inhibin-alpha subunit is an independent prognostic parameter in human endometrial carcinomas: analysis of inhibin/activinalpha, -betaA and -betaB subunits in 302 cases. Eur J Cancer. 2009;45(7): 1304-14.

20. Florio P, Luisi S, D'Antona D, Severi FM, Rago G, Petraglia F. Maternal serum inhibin A levels may predict pregnancy outcome in women with threatened abortion. Fertil Steril. 2004;81(2): 468-470.

21. Segal S, Gor H, Correa N, Mercado R, Veenstra K, Rivnay B. Inhibin A: marker for diagnosis of ectopic and early abnormal pregnancies. Reprod Biomed Online. 2008;17(6):789-94.
22. Johns J, Muttukrishna S, Lygnos M, Groome N, Jauniaux E. Maternal serum hormone concentrations for prediction of adverse outcome in threatened miscarriage. Reprod Biomed Online. 2007; 15(4):413-21.

23. Al-Azemi M, Ledger WL, Diejomaoh M, Mousa M, Makhseed M, Omu A. Measurement of inhibin A and inhibin pro-alphaC in early human pregnancy and their role in the prediction of pregnancy outcome in patients with recurrent pregnancy loss. Fertil Steril. 2003;80(6): 1473-9.

24. Prakash A, Laird S, Tuckerman E, Li TC, Ledger WL. Inhibin A and activin $A$ may be used to predict pregnancy outcome in women with recurrent miscarriage. Fertil Steril. 2005;83(6):1758-63.

25. Pautier $P$, Ghione S, Brailly-Tabard S, Lhommé C, Morice P, Bidart JM. Are serum inhibin concentrations new markers of placental tumours in the course of chemotherapy? Hum Reprod. $2001 ; 16(11): 2434-7$.

26. Florio $P$, Severi FM, Cobellis $L$, Danero $S$, Bomè $A$, Luisi $S$, et al. Serum activin $A$ and inhibin A. New clinical markers for hydatidiform mole. Cancer. 2002;94(10):2618-22.

27. Seifer DB, Lambert-Messerlian GM, Canick JA, Frishman GN, Schneyer AL. Serum inhibin levels are lower in ectopic than intrauterine spontaneously conceived pregnancies. Fertil Steril. 1996;65(3):667-9.

28. Kirk E, Papageorghiou AT, Van Calster B, Condous G, Cowans $N$, Van Huffel $S$, et al. The use of serum inhibin $A$ and activin A levels in predicting the outcome of 'pregnancies of unknown location'. Hum Reprod. 2009;24(10):2451-6.

29. Malone FD, Canick JA, Ball RH, Nyberg DA, Comstock CH, Bukowski $R$, et al. First-trimester or second-trimester screening, or both, for Down's syndrome. N Engl J Med. 2005;353(19):2001-11.

30. Ramos-Corpas DJ, Santiago JC. Combined test + inhibin A at week 13 in contingent sequential testing: an interesting alternative for first-trimester prenatal screening for Down syndrome. Prenat Diagn. 2008;28(9):833-8.

31. Paiwattananupant K, Phupong V. Serum inhibin A level in preeclampsia and normotensive pregnancy. Hypertens Pregnancy. 2008;27(4):337-43.

32. Kang JH, Farina A, Park JH, Kim SH, Kim JY, Rizzo N, et al. Down syndrome biochemical markers and screening for preeclampsia at first and second trimester: correlation with the week of onset and the severity. Prenat Diagn. 2008;28(8):704-9.

33. Phupong V, Paiwattananupant K, Honsawek S. Inhibin A levels and severity of preeclampsia. Arch Gynecol Obstet. 2009;280(2): 183-6.

34. Salomon L, Benattar C, Audibert F, Fernandez H, Duyme M, Taieb $J$, et al. Severe preeclampsia is associated with high inhibin $A$ levels and normal leptin levels at 7 to 13 weeks into pregnancy. Am J Obstet Gynecol. 2003;189(6):1517-22.

35. Spencer K, Cowans NJ, Nicolaides KH. Maternal serum inhibin-A and activin-A levels in the first trimester of pregnancies developing pre-eclampsia. Ultrasound Obstet Gynecol. 2008;32(5):622-6.

36. Sibai BM, Koch MA, Freire S, Pinto e Silva JL, Rudge MV, Martins-Costa $S$, et al. Serum inhibin A and angiogenic factor levels in pregnancies with previous preeclampsia and/or chronic hypertension: are they useful markers for prediction of subsequent preeclampsia? Am J Obstet Gynecol. 2008;199(3):268.e 1-9.

37. Florio P, Reis FM, Pezzani I, Luisi S, Severi FM, Petraglia F. The addition of activin $A$ and inhibin $A$ measurement to uterine artery Doppler velocimetry to improve the early prediction of pre-eclampsia. Ultrasound Obstet Gynecol. 2003;21 (2):165-9. 